\title{
EFFECT OF PARTICLE SIZE ON THE MINIMUM TRANSPORT VELOCITY FOR HORIZONTAL PNEUMATIC CONVEYING OF SOLIDS
}

\author{
SHIGERU MATSUMOTO, MAKOTO KIKUTA \\ AND SIRO MAEDA \\ Department of Chemical Engineering, Tohoku University, \\ Sendai 980
}

\begin{abstract}
The minimum air velocities required to keep particles from depositing on the bottom of a pipe are measured experimentally for 19 groups of spherical particles ranging from 20 to 1600 microns in three pipes of 20, 26 and $49 \mathrm{~mm}$ I.D. It is found that there is a substantial difference in the effect of particle size between fine- and coarse-particle suspensions. This difference is explained theoretically by means of numerical simulation on a digital computer based on the previously proposed ellipsoid bouncing model. A general correlation is presented to predict the minimum transport velocity over a wide range of particle size, in which an empirical equation for the distinction between the fine- and the coarse-particles system is included.
\end{abstract}

\section{Introduction}

In the design of a pneumatic conveying system for particulate solids, the most fundamental problem is the prediction of the minimum transport velocity. This is defined as the minimum superficial air velocity required for the transport of solids without the settling or formation of stationary bed on the bottom of a horizontal pipe. Despite several previous articles $^{1-3,11-15\rangle}$ on this subject, no satisfactory general correlation has been developed because each experiment was performed for a narrow range of parameters. These investigations are summarized in a review by Duckworth ${ }^{4}$.

We previously presented empirical correlations for the minimum transport velocity and the saltation velocity of granular particles greater than about 300 microns $^{8,9}$.

In this paper, we are concerned with the minimum transport velocity for horizontal pneumatic conveying of solids over a wide range of particle size from 20 to 1600 microns. The objectives of the present investigations are to clarify the effect of particle size on the minimum transport velocity and to establish a general correlation applicable to practical operating conditions.

\section{Experimental Apparatus and Procedures}

Experimental apparatus and procedures in this investigation are basically the same as those in the

Received October 1, 1976. Correspondence concerning this article should be addressed to S. Matsumoto. M. Kikuta is now with Kansai Paint Co., Ltd., Hiratsuka 254. previous paper ${ }^{8}$. Test sections were $26 \mathrm{~m}$ long Pyrex glass pipes of 20, 26 and $49 \mathrm{~mm}$ I.D. The experiments were carried out by reducing the superficial air velocity at a constant solids flow rate until the deposition of the particles could be seen by visual inspection.

Spherical particles, after being sieved with Tyler standard screens, were used in this investigation. The physical properties of the solids are tabulated in Table 1. The particle size was determined from microscope measurements on about 1000 particles, and the median value in the particle-size distribution was taken as representative.

Table 1 Properties of solid particles

\begin{tabular}{|c|c|c|c|c|c|c|}
\hline Materials & $\begin{array}{c}\rho_{p} \\
{\left[\mathrm{~g} / \mathrm{cm}^{3}\right]}\end{array}$ & $\begin{array}{l}d_{p} \\
{[\mu]}\end{array}$ & $\begin{array}{c}\text { Coefficient } \\
\text { of varia- } \\
\text { tion }[-]\end{array}$ & $\begin{array}{c}u_{t} \\
{[\mathrm{~m} / \mathrm{sec}]}\end{array}$ & $\begin{array}{l}\operatorname{Re}_{p} \\
{[-]}\end{array}$ & $\begin{array}{l}F r_{p} \\
{[-]}\end{array}$ \\
\hline \multirow[t]{13}{*}{ Glass } & \multirow[t]{13}{*}{2.5} & 65 & 0.218 & 0.33 & 1.4 & 13.1 \\
\hline & & 75 & 0.182 & 0.43 & 2.1 & 15.9 \\
\hline & & 87 & 0.100 & 0.59 & 3.4 & 20.2 \\
\hline & & 99 & 0.189 & 0.77 & 5.1 & 24.8 \\
\hline & & 110 & 0.149 & 0.91 & 6.7 & 27.7 \\
\hline & & 129 & 0.138 & 1.01 & 8.8 & 28.3 \\
\hline & & 163 & 0.121 & 1.26 & 13.6 & 31.5 \\
\hline & & 174 & 0.164 & 1.32 & 15.0 & 32.3 \\
\hline & & 266 & 0.124 & 2.06 & 36.5 & 40.3 \\
\hline & & 311 & 0.153 & 2.42 & 50.2 & 43.8 \\
\hline & & 480 & 0.102 & 3.74 & 120 & 54.1 \\
\hline & & 1020 & 0.091 & 7.74 & 526 & 77.4 \\
\hline & & 1640 & 0.111 & 9.82 & 1070 & 77.4 \\
\hline \multirow[t]{6}{*}{ Copper } & \multirow[t]{6}{*}{8.7} & 20 & 0.192 & 0.18 & 0.24 & 11.1 \\
\hline & & 55 & 0.193 & 0.85 & 3.1 & 36.4 \\
\hline & & 97 & 0.134 & 1.72 & 11.1 & 56.1 \\
\hline & & 297 & 0.077 & 5.36 & 106 & 98.9 \\
\hline & & 522 & 0.159 & 9.29 & 323 & 130 \\
\hline & & 758 & 0.154 & 12.5 & 630 & 145 \\
\hline
\end{tabular}




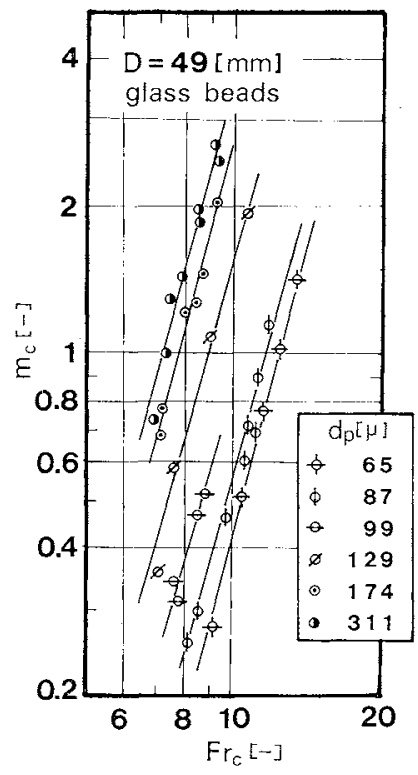

Fig. 1 Typical plots on logarithmic coordinates of mass flow ratio versus Froude number at the minimum-transport condition for fine-particles

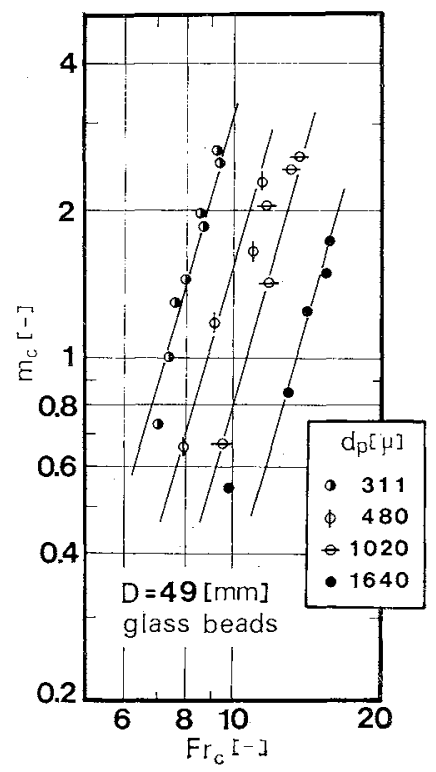

Fig. 2 Typical plots on logarithmic coordinates of mass flow ratio versus Froude number at the minimum-transport condition for coarse-particles

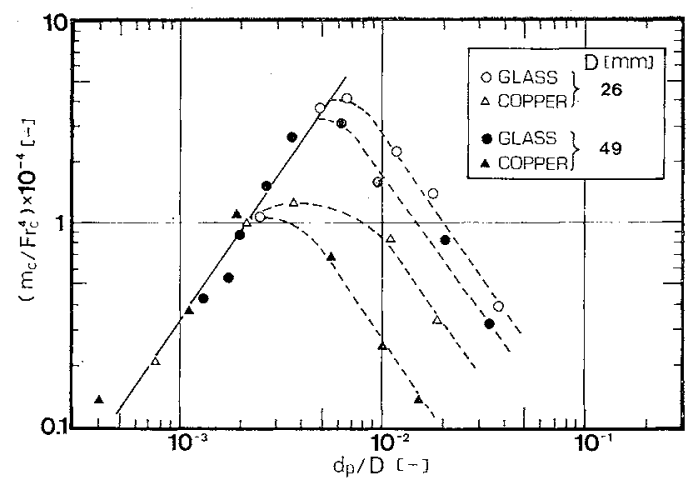

Fig. 3 Characteristic conveyabilities of material as function of particle to pipe size ratio

\section{Experimental Results}

In Fig. 1, typical plots are shown of mass flow ratio versus Froude number at the minimum-transport condition for fine particles of glass in the $49 \mathrm{~mm}$ I.D. pipe. Experimental results for coarse particles of glass are shown in Fig. 2 for comparison. From these figures, it is found that all experiments can be approximately correlated by $m_{c} \propto F r_{c}^{4}$. However, it is also found that the dependence of minimum transport velocity on the particle size in the fine-particles system is completely contrary to that in the coarse-particles system. That is, the smaller the particle size, the greater the superficial air velocity that will be required to maintain a given solids rate for the fine-particles system. On the other hand, the required air velocity will decrease with decreasing particle size in the coarse-particles system. The same feature was also obtained for other experimental conditions.

Similar results are reported by $\mathrm{Zenz}^{15)}$ and $\mathrm{Halow}^{5)}$. Zenz examined the conveyability of single particles, and pointed out that the saltation velocity may increase with decrease in particle size when the size falls below a certain limit. Halow also reported that there existed a minimum, at a particular size of microspheroidal catalyst, in the gas velocity required to initiate sliding or rolling of single particles in turbulent horizontal pipe flow.

Since the relation $m_{c} \propto F r_{c}^{4}$ is valid for almost all the particle size, a quantity $m_{c} / F_{c}^{4}$ will be independent of operating variables, depending on the properties of solids and/or conduit. The quantity $m_{c} / F r_{c}^{4}$, in other words, represents a characteristic saturation carrying capacity of the air flow. In what follows, therefore, this quantity is termed the characteristic conveyability of material. The characteristic conveyabilities are presented in Fig. 3 as function of particle to pipe diameter ratio. It can be seen that the conveyability has a maximum at a certain value of $d_{p} / D$, and maximum is virtually dependent only upon the species of solid.

The particle diameter providing the maximum conveyability, expressed as $d_{p}^{*}$, was correlated by

$$
d_{p}^{*} / D=1.39\left(\rho_{p} / \rho_{a}\right)^{-0.74}
$$

As a result, solid particles may be classified into two groups at the critical diameter $d_{p}^{*}$ from the viewpoint of this coveyability: one referred to as the fine particles and the other as the coarse particles.

It will be also seen from Fig. 3 that the conveyability of fine particles may be correlated by $d_{p} / D$ alone, whereas for coarse particles it is dependent on both the particle density and size (Eq. (3)). As shown in Fig. 4, the following correlation was obtained:

$$
m_{c}=5.56 \times 10^{3}\left(d_{p} / D\right)^{1.43}\left(F r_{c} / 10\right)^{4}
$$

where the powers and factor were determined by the 
least-squares method. All the points are seen to lie within $\pm 50 \%$.

Finally, it will be concluded that the minimum transport velocity of fine particles with a diameter smaller than $d_{p}^{*}$ can be calculated by Eq. (2), and that of coarse particles larger than $d_{p}^{*}$ can be calculated by the following equation, presented previously ${ }^{8)}$;

$$
m_{c}=0.373\left(\rho_{p} / \rho_{a}\right)^{1.08}\left(F r_{p} / 10\right)^{-3.7}\left(F r_{c} / 10\right)^{3.61}
$$

\section{Theoretical Consideration}

3. 1 Simulation based on the ellipsoid bouncing model

We previously presented the ellipsoid bouncing model to simulate the irregular bounce of particles on the duct wall ${ }^{7}$. In this stochastic model, a particle was approximated by an ellipsoid, and two-dimensional bouncing motion was assumed on a flat plate. The motion of particles in a rectangular or circular conduit was satisfactorily simulated by the Monte Carlo method for the model on a digital computer ${ }^{7,10)}$.

In this article, the same model is also used in order to see the effect of particle size on the minimum transport velocity. To simplify the analysis, we use the two-dimensional model assuming a rectangular duct as the conduit, though the actual conduit is a circular pipe. By this simplification, the effect of particle size on the bouncing or flowing of particles in the conduit becomes clearer than in the three-dimensional circular pipe model. In the Monte Carlo simulation, the minimum air velocity required to keep particles from depositing on the bottom of the duct is determined by reducing gradually the air velocity in much the same way as in the experiment.

Examples of simulated results are shown in Fig. 5, which indicates the effect of air velocity upon the concentration distribution of 500-micron glass beads. It can be seen from this figure that the concentration distribution may be approximated by the following exponential function;

$$
n_{i} / n_{0}=c \cdot \exp (-\gamma y)
$$

As the air velocity is reduced, the particles become more concentrated near the bottom, which lowers the slope of the lines in Fig. 5, namely $\gamma$ in Eq. (4). If the air velocity is further reduced to a particular value, the particles finally begin to settle out of suspension. Since it takes much computational effort to determine the limit directly by simulation, it is estimated by the following extrapolation method of the concentration gradient.

Whenever almost all the particles begin settling down on the bottom, the gradient $\gamma$ becomes so small as to be the order of $10^{-1}$. Hence, the air velocity at $\gamma=0.1$ is used as the approximate value of the minimum transport velocity. This is determined from the plots of $\gamma$ versus $u_{a}$ as shown in Fig. 6, and written as

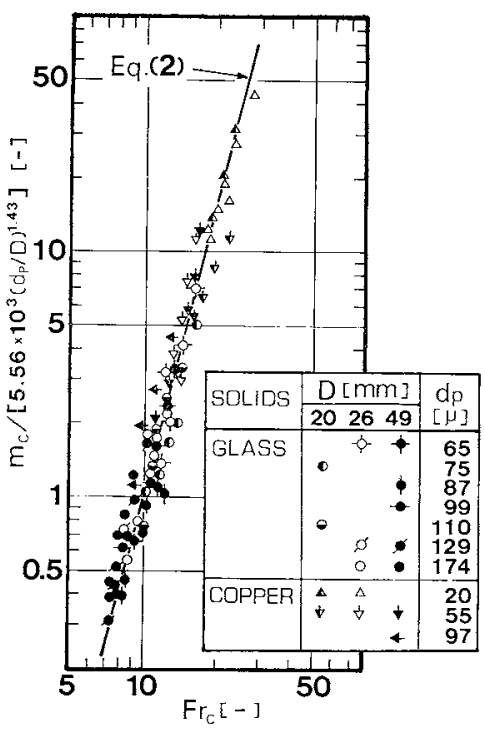

Fig. 4 Correlation of mass flow ratio with Froude number at the minimum-transport condition

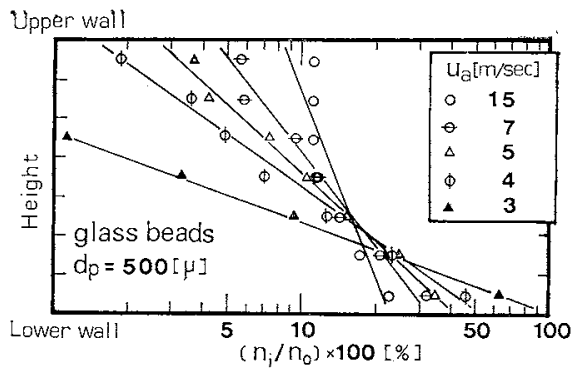

(Height of duct $=26 \mathrm{~mm}, \eta=1.02$ )

Fig 5 Effect of air velocity on the concentration distribution of particles obtained by simulation

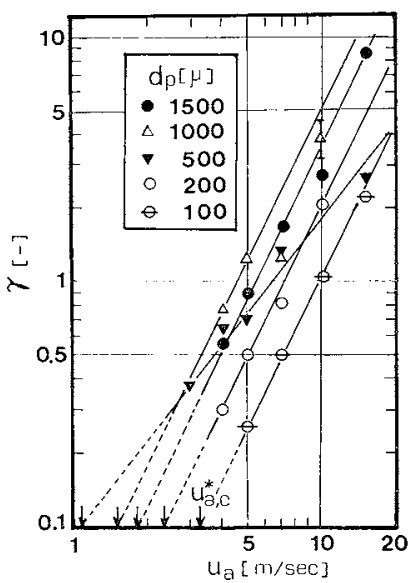

The set of parameters is the same as in Fig. 5 .

Fig. 6 Plots of gradient of concentration distribution versus air velocity for glass beads

$u_{a, c}^{*}$. This will represent the minimum transport velocity of a single particle or extremely dilute suspension, because the interactions of particles are not taken into account in the model. As this velocity is peculiar to particles, it will be termed the characteristic 


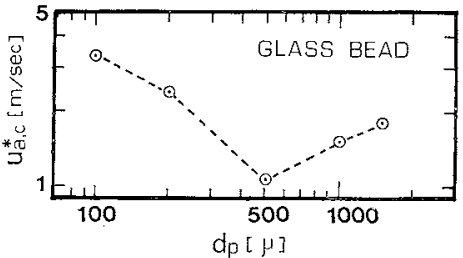

The set of parameters is the same as in Fig. 5.

Fig. 7 Characteristic minimum transport velocity of glass beads as function of particle size

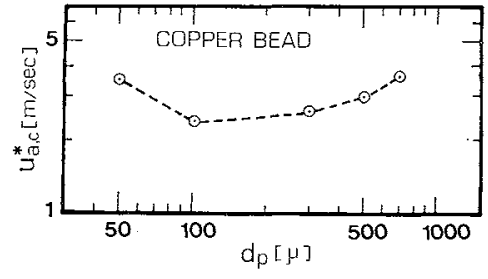

(Height of duct $=26 \mathrm{~mm}, \eta=8.03$ )

Fig. 8 Characteristic minimum transport velocity of copper beads as function of particle size

minimum transport velocity in this article.

In Fig. 7, the characteristic minimum transport velocities of glass beads are plotted against the particle size. This figure shows that $u_{a, c}^{*}$ takes a minimum at a diameter of 500 microns. Hence, it will be expected that the maximum conveyability occurs at $d_{p}=500$ microns. This feature is identical with that in the experiments, though the diameter providing the maximum is not exactly equal to any in the experiments. In the case of the data obtained for the $26 \mathrm{~mm}$ pipe, the maximum conveyability of glass appears at a diameter of 200 microns or so, while it appears at 500 microns in the simulation (Fig. 7). This difference is considered to be caused by the fact that the simulation model is two-dimensional.

As for the copper beads, the calculated $u_{a, c}^{*}$ shows a minimum at $d_{p}=100$ microns, as shown in Fig. 8 .

The above simulation may confirm the conclusion that there exists a maximum in the characteristic conveyability at a particular size. However, from what has been described in the above, we cannot know how the particle size is associated with that feature. To make the mechanism clear, then, we examined the bouncing characteristics and the state of motion of particles in a duct, as described in the following section.

\section{2 Dissipation coefficient of kinetic energy of particle}

The phenomena of particle deposition and blockage will mean that the particles, through repeated colli-

\footnotetext{
* $\Delta E$ is given by$$
\Delta E=\frac{1}{2}\left(-\frac{\pi}{6}-d_{p}^{3} \rho_{p}\right)\left[\left(u_{p}^{\circ 2}+v_{p}^{\circ 2}+k^{2} \omega_{p}^{\circ 2}\right)-\left(u_{p}^{\prime 2}+v_{p}^{\prime 2}+k^{2} \omega_{p}^{\prime 2}\right)\right]
$$

** Even if a particle is an ellipsoid, Eq. (10) can be used when the sphericity is closer to unity.
}

sions with the duct wall, dissipate the kinetic energy supplied by the fluid, resulting in their falling on the bottom of the conduit. We introduce a concept of dissipation coefficient of kinetic energy of particle, to illustrate this problem.

The dissipation coefficient, $\Phi$, is defined as the ratio of dissipated energy owing to the sequential collisions per unit length of duct, $\Delta E$, to kinetic energy of the particle in the air stream, $E_{0}$. The dissipated energy $\Delta E$ is given by

$$
\Delta E=\sum_{i=1}^{N e} \Delta E_{i}
$$

where $\Delta E_{i}$ is the dissipated energy during each bounce and $N_{c}$ is the number of collisions in a unit length of duct. The average value of kinetic energy before collision $E_{0}$ is considered to be practically constant as the dissipated energy during bounce is supplied by the air stream through the flight. The dissipation coefficient $\Phi$, then, becomes

$$
\Phi=\Delta E / E_{0}=\left(\sum_{i=1}^{N_{e}} \Delta E_{i}\right) / E_{0}
$$

If we suppose the dissipated energy $\Delta E_{i}$ to remain virtually constant for all collisions, written as $(\Delta E)_{\mathrm{av}}$, Eq. (6) is approximated by

$$
\Phi \doteqdot N_{c} \times(\Delta E)_{\text {av }} / E_{0}
$$

This expression may also be written in the alternative form

$$
\Phi \fallingdotseq N_{\mathrm{c}} \times\left(\Delta E / E_{0}\right)_{\mathrm{av}}
$$

It corresponds to the degree of required energy for the transport of a single particle by the air flow, and is in a sense closely related to the characteristic conveyability of particle.

Let $\left(u_{p}^{\circ}, v_{p}^{\circ}, \omega_{p}^{\circ}\right)$ and $\left(u_{p}^{\prime}, v_{p}^{\prime}, \omega_{p}^{\prime}\right)$ denote the velocity components of a particle before and after bounce, respectively. The fraction of dissipated energy owing to one bounce is given by

$$
\frac{\Delta E}{E_{0}}=1-\frac{\left(u_{p}^{\prime} / u_{p}^{\circ}\right)^{2}+\left(e_{u} \tan \theta_{i}\right)^{2}+k^{2}\left(\omega_{p}^{\circ} / u_{p}^{\circ}\right)^{2}\left(\omega_{p}^{\prime} / \omega_{p}^{\circ}\right)^{2}}{1+\left(\tan \theta_{i}\right)^{2}+k^{2}\left(\omega_{p}^{\circ} / u_{p}^{\circ}\right)^{2}}
$$

where $k$ is a radius of gyration and calculated, for instance, as follows:

and

$$
k=(1 / \sqrt{10}) d_{p} \quad \text { for sphere }
$$

$$
k=\sqrt{\frac{1+\eta}{5(1+\eta)^{2}}} d_{p} \quad \text { for ellipsoid }
$$

When the particle is spherical, it becomes;

$$
-\frac{\Delta E}{E_{0}}=1-\frac{\left(u_{p}^{\prime} / u_{p}^{\circ}\right)^{2}+\left(e_{y} \tan \theta_{i}\right)^{2}+(2 / 5) \zeta^{2}\left(\omega_{p}^{\prime} / \omega_{p}^{\circ}\right)^{2}}{1+\left(\tan \theta_{i}\right)^{2}+(2 / 5) \zeta^{2}}
$$

By taking statistics in the simulation, we examined these variables, $\Phi, N_{o}$ and $\left(\Delta E / E_{0}\right)_{\mathrm{a}, \mathrm{r}}$, and the parameters contained in these variables. Simulations were 


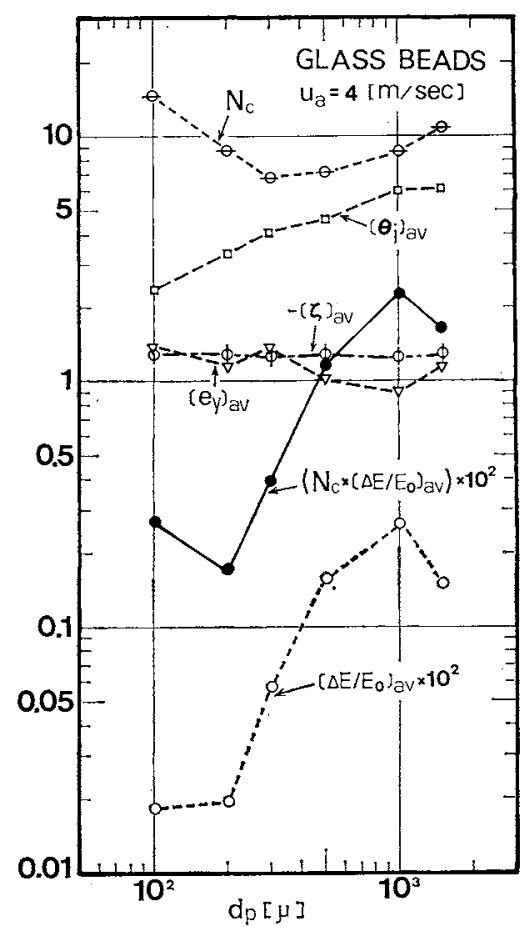

(Height of duct $=26 \mathrm{~mm}, \eta=1.02$ )

Fig. 9 Various variables in the simulation as function of particle size for glass beads

carried out at several air velocities approaching the minimum transport velocity. Examples of computed results for glass beads at $u_{a}=4 \mathrm{~m} / \mathrm{sec}$ are shown in Fig. 9. This figure shows that $\left(\Delta E / E_{0}\right)_{\mathrm{av}}$ is virtually unchanged between $d_{p}=100$ and 200 microns but increases rapidly as the diameter increases further. The number of collisions has a minimum at a diameter of 300 or 500 microns. Consequently, the product of $\left(\Delta E / E_{0}\right)_{a \nabla}$ and $N_{c}$ has a minimum at $d_{p}=200$ microns as shown in Fig. 9. This result is in good agreement with the experiments in which the 200-micron glass beads have a maximum in their characteristic conveyability in the $26 \mathrm{~mm}$ I.D. pipe.

Average values of $\zeta, e_{y}$ and $\theta_{i}$ are also shown in Fig. 9. The incident angle $\left(\theta_{i}\right)_{\mathrm{av}}$ increases monotonically with increase in the particle size, while both $\left(e_{y}\right)_{\mathrm{a} \nabla}$ and $(\zeta)_{\mathrm{av}}$ indicate no appreciable tendency as for the effect of particle size. Moreover, statistical results of velocity ratios between after and before bounce, $\left(u_{p}^{\prime} / u_{p}^{\circ}\right)_{\mathrm{av}}$ and $\left(\omega_{p}^{\prime} / \omega_{p}^{\circ}\right)_{\mathrm{av}}$, which are not illustrated in the figure, are scarcely dependent on the particle size. It is interesting to note that the number of collisions has a minimum for particles sized somewhere between the 300 and 500 microns as shown in Fig. 9. The reason for this seems to be the net effect of gravity and aerodynamic drag force acting on the particle. This problem will be discussed from another viewpoint in the next section.

Figure 10 shows the results for copper beads when $u_{a}=4 \mathrm{~m} / \mathrm{sec}$. In this case, the $\left(\Delta E / E_{0}\right)_{\text {av }}$ and the

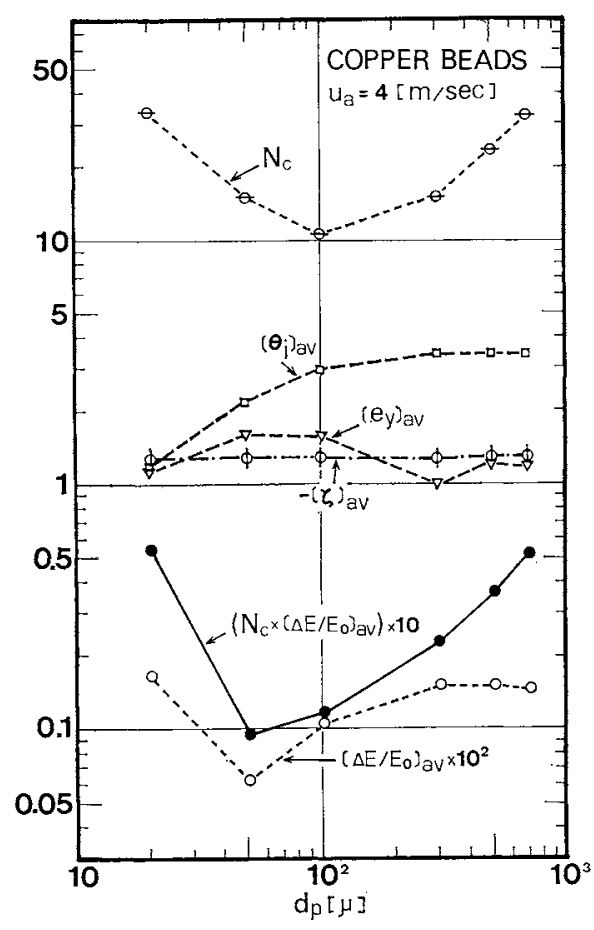

(Height of duct $=26 \mathrm{~mm}, \eta=1.03$ )

Fig. 10 Various variables in the simulation as function of particle size for copper beads

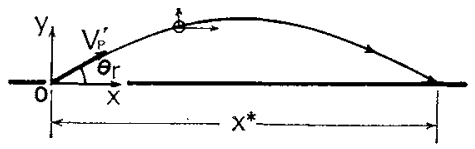

Fig. 11 Model for calculation of the leap length of particle

product $N_{c} \times\left(\Delta E / E_{0}\right)_{\mathrm{a} v}$ shows minima at $d_{p}=50$ microns, while the minimum $N_{c}$ occurs at $d_{p}=100$ microns.

In the simulation at an air velocity of $5 \mathrm{~m} / \mathrm{sec}$, similar results were obtained for both glass and copper beads, though they are not shown in figures. We were much interested in minimum values of $\left(\Delta E / E_{0}\right)_{\mathrm{av}}$, and discussed this in its various aspects. Nevertheless, we could not obtain a clear explanation because many factors were combined in a complicated way.

\section{3 Effect of particle size on the collision number}

To clarify the reason why the number of collisions has a minimum at a particular size under condition of constant air velocity, we examined how far a single particle could travel in the air stream after bounce, by making a simplified model.

As illustrated in Fig. 11, a spherical particle with a diameter of $d_{p}$ rebounds from the surface with an initial velocity of $V_{p}^{\prime}$ and at a reflection angle of $\theta_{r}$, and falls again on the surface after travelling a distance $x^{*}$. This distance $x^{*}$ is termed the leap length of particle. Several assumptions and equations for calculation of $x^{*}$ are described in the Appendix.

Figure 12 shows the calculated results of the re- 


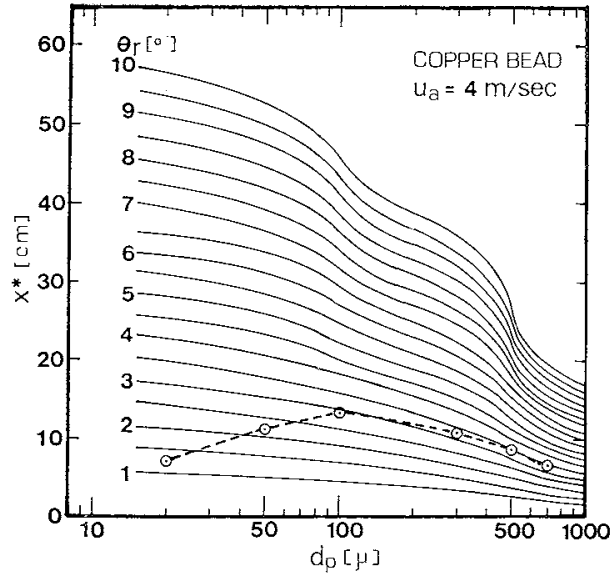

Fig. 12 Leap length of particle as function of particle size with reflection angle as parameter for copper beads

lations between $x^{*}$ and $d_{p}$ for copper beads with the parameters of $\theta_{r}$ at an air velocity of $4 \mathrm{~m} / \mathrm{sec}$. The reflection angle $\theta_{r}$ is closely related to the incident angle $\theta_{i}$, and may be considered to be proportional to $\theta_{i}$. Letting $\theta_{i}$ be roughly $\theta_{r}$ for convenience, the relationship between $x^{*}$ and $d_{p}$ is illustrated as a dotted line in Fig. 12. This curve indicates a maximum at $d_{p}=100$ microns. Since $N_{c}$ is inverse to $x^{*}$, this result is consistent with the tendency of $N_{c}$ obtained in the simulation. This will be explained as follows: As the incident angle increases with increase in particle size at a constant air velocity, the reflection angle also increases with increase in particle size. Hence, the leap length $x^{*}$ tends to lengthen with increase in particle size. On the other hand, both the effect of aerodynamic drag force exerted on the particle, and the initial velocity of particle, decrease as the particle size increases*. In consequence, the leap length is reduced when the particle size exceeds a certain limit, thus having a minimum value.

For the case of glass beads and other air velocities, similar results were obtained.

\section{Conclusions}

(1) The effect of particle size upon the minimum transport velocity for fine-particle suspension is completely contrary to that for coarse-particles. Hence, the characteristic conveyability of solids has a maximum at a particular size of solid.

(2) For fine-particle suspensions, the minimum transport velocity can be satisfactorily correlated by Eq. (2). The boundary of distinction between the fine- and the coarse-particles is defined by Eq. (1).

(3) The contribution of particle size to the minimum transport velocity was confirmed in the simulation based on the ellipsoid bouncing model. The mechanism of this phenomenon was explained by

\footnotetext{
* These effects are obvious from Eqs. (A-1) and (A-8).
}

introducing a concept of dissipation coefficient of kinetic energy of particle. From both the simulation and simplified analytical model, it was proved that the number of collisions of particles with the wall has a minimum at a particular size of solid, under condition of constant air velocity.

It is considered that the complicated behaviour of the particles is caused by the combined effects of the several factors mentioned above.

\section{Appendix}

The following assumptions are used in the construction of the model:

1) A particle is spherical.

2) Only gravitational force acts on the particle in the $y$ direction, assuming that both drag and lift force are negligible.

3) An air velocity profile is uniform.

Under these assumptions, equations of motion for a single particle are described by:

$$
\begin{aligned}
& \frac{d^{2} x}{d t^{2}}=\frac{3}{4} \frac{\rho_{a}}{\rho_{p}}-\frac{1}{d_{p}} C_{D} \cdot\left(u_{a}-\frac{d x}{d t}-\right)^{2} \\
& d^{2} y / d t^{2}=-g
\end{aligned}
$$

The initial conditions are:

$$
t=0: x=y=0 ; \frac{d x}{d t}=V_{p}^{\prime} \cos \theta_{r}=u_{p}^{\prime}, \frac{d y}{d t}=V_{p}^{\prime} \sin \theta_{r}
$$

From these equations, the solutions for the leap length are obtained, for the following three regions of drag coefficient, as: a) Stokes' law region;

$$
\frac{x^{*}}{D}=2 K_{1} F r^{2} \sin \theta_{r}-\frac{1-K_{1} \cos \theta_{r}}{K_{0}}\left[1-\exp \left(-2 K_{0} K_{1} F r^{2} \sin \theta_{r}\right)\right]
$$

b) Allen's law region;

$$
\begin{aligned}
\frac{x^{*}}{D}= & 2 K_{1} F r^{2} \sin \theta_{r}-\frac{2 \sqrt{1-} K_{1} \cos \theta_{r}}{K_{2}} \\
& \times\left[1-\left(1+K_{1} K_{2} F r^{2} \sin \theta_{r} \sqrt{1-K_{1} \cos \theta_{r}}\right)^{-1}\right]
\end{aligned}
$$

c) Newton's law region;

$$
\frac{x^{*}}{D}=2 K_{1} F r^{2} \sin \theta_{r}-\frac{1}{K_{3}} \ln \left[1+2 K_{1} K_{3} F r^{2} \sin \theta_{r}\left(1-K_{1} \cos \theta_{r}\right)\right]
$$

where

$$
\begin{aligned}
& K_{0}=18 \mu_{a} D / \rho_{p} d_{p}^{2} u_{a}, \quad K_{1}=\left(u_{p}^{\prime} / u_{a}\right)\left(1 / \cos \theta_{r}\right), \\
& K_{2}=\frac{15}{2} \frac{\rho_{a}}{\rho_{p}}\left(\frac{D}{d_{p}}\right)^{1.5} R e^{-0.5}, \quad K_{3}=0.33 \frac{\rho_{a}}{\rho_{p}} \frac{D}{d_{p}}
\end{aligned}
$$

The initial velocity of particle is required for the numerical evaluation of the above solutions. We estimate the component of initial velocity, $u_{p}^{\prime}$, by Konno's empirical equation ${ }^{6)}$;

$$
u_{p}^{\prime} / u_{a}=1-1.35 \times 10^{-3} / D\left(u_{t} / \sqrt{g D}\right)^{0.65} \quad(D:[\mathrm{m}])
$$

Substituting this equation into Eq. (A-7), we can evaluate the leap length $x^{*}$ with the parameter of $\theta_{r}$ for a given condition.

\section{Nomenclature}

$\begin{array}{llr}C_{D} & =\text { drag coefficient } & {[-]} \\ c & =\text { constant in Eq. (4) } & {[-]} \\ D & =\text { pipe diameter } & {[\mathrm{m}]} \\ d_{p} & =\text { particle diameter } & {[\mathrm{m}]} \\ d_{p}^{*} & =\text { particle diameter providing the maximum } & \\ & & \text { conveyability } \\ E_{0} & = & \text { kinetic energy of particle before collision }\end{array}$ 


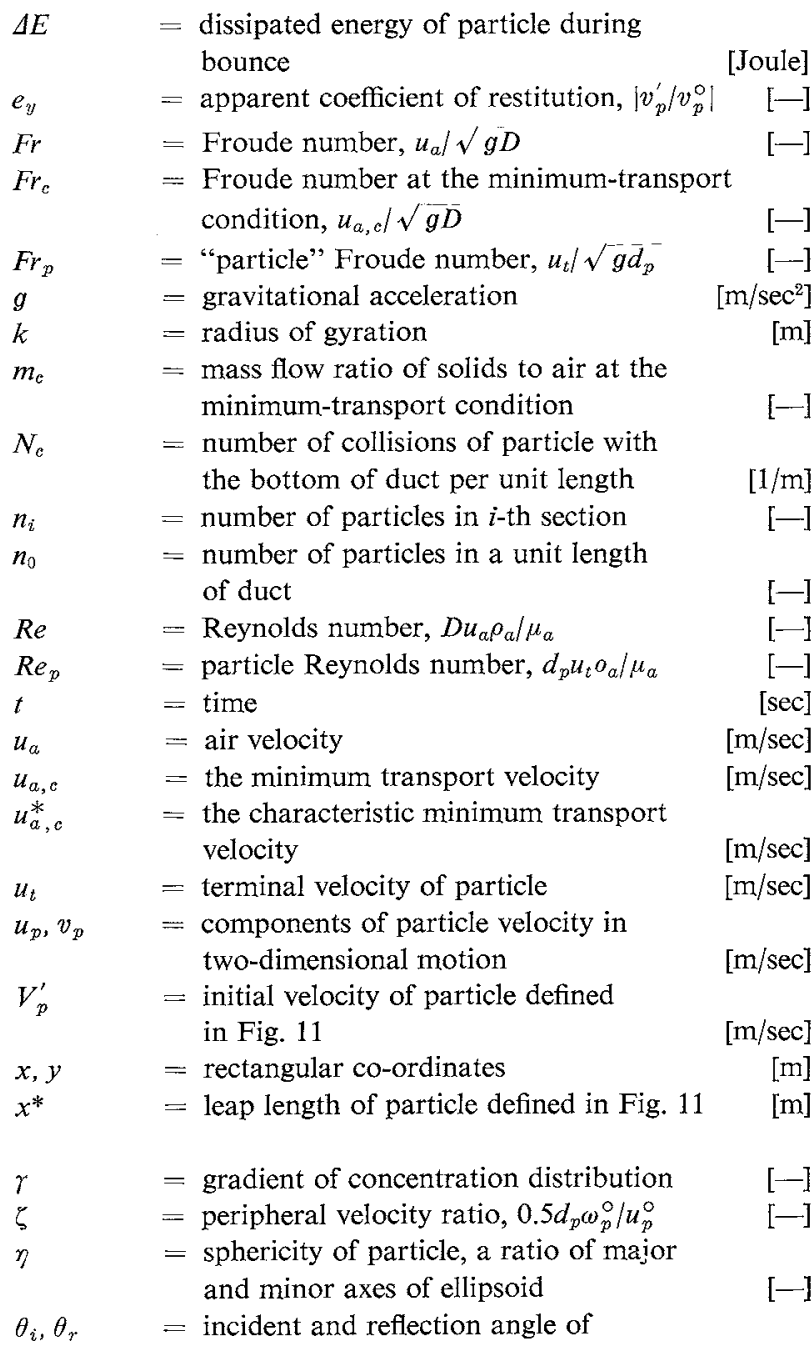

particle, respectively

[degrees]

$=$ viscosity of air

$\mu_{a}$

$=$ density of air and

$=$ dissipation coefficient of kinetic energy

of particle

$[\mathrm{kg} / \mathrm{m} \cdot \mathrm{sec}]$

$\Phi$

$=$ angular velocity of particle

$\omega_{p}$

$\langle$ Superscripts〉

- $=$ before bounce

, $\quad=$ after bounce

\section{Literature Cited}

1) Barth, W.: Chem-Ing-Tech., 26, 29 (1954).

2) Bohnet, M.: VDI-Forschungsheft, 507 (1965).

-] 3) Doig, I. D. and G. H. Roper: Australian Chem. Eng., 4, 9 (1963).

$[-]$

$[-]$

$[-]$

[sec]

$[\mathrm{m} / \mathrm{sec}]$

$[\mathrm{m} / \mathrm{sec}]$

$[\mathrm{m} / \mathrm{sec}]$

$[\mathrm{m} / \mathrm{sec}]$

$[\mathrm{m} / \mathrm{sec}]$

$[\mathrm{m} / \mathrm{sec}]$

[m]

[m]

$[-]$

$[-]$

$[-]$

4) Duckworth, R. A.: Proceedings of 1st International Conference on the Pneumatic Transport of Solids in Pipes, BHRA, Bedford, England, R2-25 (1971).

5) Halow, J. S.: Chem. Eng. Sci., 28, 1 (1973).

6) Konno, H., S. Saito and S. Maeda: Kagaku Kögaku, 31, 243 (1967).

7) Matsumoto, S. and S. Saito: J. Chem. Eng. Japan, 3, 83 (1970).

8) Matsumoto, S., M. Hara, S. Saito and S. Maeda: ibid., 7, 425 (1974).

9) Matsumoto, S., S. Harada, S. Saito and S. Maeda: ibid., 8, 331 (1975).

10) Matsumoto, S., S. Saito and S. Maeda: ibid., 9, 23 (1976).

11) Patterson, R. C.: Trans. ASME, J. Eng. for Power, 81, 43 (1959).

12) Rose, H. E. and R. A. Duckworth: Proceedings of the I. Chem. E.-VTG/VDI Joint Meetings, p. 53 (1968).

13) Thomas, D. G.: AIChE J., 8, 373 (1962).

14) Zenz, F. A. and D. F. Othmer: "Fluidization and FluidParticle Systems", Reinhold, New York (1960).

15) Zenz, F. A.: Ind. Eng. Chem., Fundam., 3, 65 (1964). 